\title{
EXPONENTIAL STABILITY FOR ABSTRACT LINEAR AUTONOMOUS FUNCTIONAL DIFFERENTIAL EQUATIONS WITH INFINITE DELAY
}

\author{
JIN LIANG \\ Teaching and Research Section of Mathematics \\ Kunming University of Science and Technology \\ Kunming 650093, P.R. CHINA \\ FALUN HUANG \\ Department of Mathematics \\ Sichuan University \\ Chengdu 610064, P.R. CHINA \\ TWUN XIAO \\ Department of Mathematics \\ Yunnan Normal University \\ Kunming 650092, P.R. CHINA
}

(Received May 5, 1994 and in revised form September 27, 1996)

ABSTRACT. Based on our preceding paper, this note is concerned with the exponential stability of the solution semigroup for the abstract linear autonomous functional differential equation

$$
\dot{x}(t)=L\left(x_{t}\right)
$$

where $L$ is a continuous linear operator on some abstract phase space $B$ into a Banach space $E$. We prove that the solution semigroup of $(*)$ is exponentially stable if and only if the fundamental operator (*) is exponentially stable and the phase space $B$ is an exponentially fading memory space.

KEY WORDS AND PHRASES: Exponentially stable, solution semigroup, abstract linear autonomous functional differential equation, fundamental operator, fading memory space.

1991 AMS SUBJECT CLASSIFICATION CODES: Primary 34K20, 34G20.

\section{INTRODUCTION}

Let $E$ be a Banach space. For an $E$-valued function $x$ defined on $(-\infty, a]$, by $x_{t}$ we denote the function $x_{t}(\theta)=x(t+\theta),-\infty<\theta \leq 0$. We consider the abstract linear autonomous functional differential equation with infinite delay

$$
\left\{\begin{array}{l}
\dot{x}(t)=L\left(x_{t}\right), \quad t>0, \\
x(\theta)=\phi(\theta), \quad \theta \in(-\infty, 0]
\end{array}\right.
$$

where $L$ is a continuous linear operator on an abstract phase space $B$ into $E$.

In [1], we have discussed some problems on (1.1), and have given a definition of the fundamental operator for (1.1) corresponding to the fundamental matrix [2,3] of $(11)$ in the case of finite-dimensional space, and some sufficient and necessary conditions of the fundamental operator of (1.1) being exponentially stable. In the present note, we investigate the exponential stability of the solution semigroup of (1.1) and set up a sufficient and necessary condition for it. It is shown that the solution semigroup of (1.1) is exponentially stable if and only if the fundamental operator of (1.1) is exponentially stable and the phase space $B$ is an exponentially fading memory space. 


\section{MAIN RESULTS}

Let $U(Y)$ be the set of all bounded linear operators from space $Y$ to $Y . \chi_{[u, v]}(\cdot)$ denotes the characteristic function of interval $[u, v]$. Suppose $B$ is a Banach space of $E$-valued functions on $(-\infty, 0]$ with a norm $\|\cdot\|_{B}$ having the following properties:

(H1). If $x:(-\infty, \alpha] \rightarrow E, a>\sigma$, is continuous on $[\sigma, a]$ and $x_{0} \in B$, then $x_{t}$ is continuous in $t \in[\sigma, a]$.

(H2). $\|\phi(0)\| \leq K\|\phi\|_{B}$ for all $\phi$ in $B$ and some constant $K$.

(H3). $\|\phi\|_{B} \leq\|\phi\|_{\beta}+\|\phi\|_{(\beta)}$ for any $\beta>0, \phi \in B$, where $\|\phi\|_{\beta}=\inf \left\{\|\psi\|_{B}: \psi \in B\right.$ and $\psi(\theta)=\phi(\theta)$ for $\theta \in(-\infty,-\beta]\},\|\phi\|_{(\beta)}=\inf \left\{\|\psi\|_{B}: \psi \in B\right.$ and $\psi(\theta)=\phi(\theta)$ for $\left.\theta \in[-\beta, 0]\right\}$.

(H4). $\|\phi\|_{\beta} \leq K_{1} \sup \{\|\phi(\theta)\|,-\beta \leq \theta \leq 0\}$ for any $\phi \in B$ and some constant $K_{1}$; sup $\|\phi\|_{\beta}$ is a locally bounded function of $\beta \geq 0$.

(H5). $\|x(\cdot)\|_{B} \leq M_{1}\|y(\cdot)\|_{B}$ for any $x, y \in B$ with $\|x(\theta)\| \leq\|y(\theta)\|$ for $\theta \in(-\infty, 0]$ and some constant $M_{1}$.

(H6). $\chi_{[-t, 0]}(\cdot) b, \chi_{(-t, 0]}(\cdot) b \in B$ for any $t \geq 0$ and $b \in E:\|\chi[0,0](\cdot) b\|_{B} \leq M\|b\|$ for $b \in E$ and some constant $M$.

$B$ is called an admissible phase space. (H4) and (H5) stem from $\left(\alpha_{3}\right),\left(\beta_{1}\right)$ and $\left(\beta_{2}\right)$ in [4]. It is clear that $[1, \mathrm{H}(3)]$ follows from (H4) and (H5). A typical example of the space $B$ could be found in [1]. It is known that for each $\phi \in B$, the solution $x(t)=x(t, \phi)$ of $(1.1)$ exists uniquely for $t \in[0, \infty)$ For every $\phi \in B, t \in[0, \infty)$, define

$$
(T(t) \phi)(\theta)=x_{t}(\theta)=x_{t}(\theta, \phi)=\left\{\begin{array}{ll}
\phi(0)+\int_{0}^{t+\theta} L\left(x_{s}\right) d s, & t+\theta>0, \\
\phi(t+\theta), & t+\theta \leq 0,
\end{array} \quad \theta \in(-\infty, 0] .\right.
$$

$\{T(t)\}_{t \geq 0}$ is called the solution semigroup of (1.1). If $L=0$, we denote by $\{S(t)\}_{t \geq 0}$ the solution semigroup, i.e.,

$$
(S(t) \phi)(0)=\left\{\begin{array}{ll}
\phi(0), & t+\theta>0, \\
\phi(t+\theta), & t+\theta \leq 0,
\end{array} \quad t \in[0, \infty), \phi \in B, \theta \in(-\infty, 0] .\right.
$$

It is also called a translation semigroup. By virtue of $(\mathrm{H} 1),\{T(t)\}_{t \geq 0}$ and $\{S(t)\}_{t \geq 0}$ are $C_{0}$ semigroups on $B$. Set

$$
\left(S_{0}(t) \phi\right)(\theta)=\left\{\begin{array}{ll}
0, & t+\theta>0, \\
\phi(t+\theta), & t+\theta \leq 0,
\end{array} \quad t \in[0, \infty), \phi \in B, \theta \dot{\in}(-\infty, 0] .\right.
$$

An admissible phase space $B$ is called to be an exponentially fading memory space, if there are constants $C, \alpha>0$ such that $\left\|S_{0}(t) \phi\right\|_{t} \leq C e^{-\alpha t}\|\phi\|_{B}$ for any $\phi \in B, t>0$. By (H1) and (H6), $S_{0}(t) \phi \in B(t>0)$. Therefore, $B$ is an exponentially fading memory space if and only if $\left\|S_{0}(t) \phi\right\|_{B} \leq C e^{-\alpha t}\|\phi\|_{B}$ for any $\phi \in B, t>0$ and some positive constants $C, \alpha$, since by (H5),

$$
\left\|S_{0}(t) \phi\right\|_{t} \leq\left\|S_{0}(t) \phi\right\|_{B} \leq M_{1}\left\|S_{0}(t) \phi\right\|_{t} \text { for any } \phi \in B, t>0 .
$$

LEMMA 1. Let $x(t, \phi)$ be the solution of (1.1). Then

$$
x(t, \phi)= \begin{cases}X(t) \phi(0)+\int_{0}^{t} X(t-\tau) L\left(S_{0}(\tau) \phi\right) d \tau, & t>0 \\ \phi(t), & t \leq 0\end{cases}
$$

or for $\theta \in(-\infty, 0]$, 


$$
\begin{aligned}
(T(t) \phi)(\theta) & = \begin{cases}X(t+\theta) \phi(0)+\int_{0}^{t+\theta} X(t+\theta-\tau) L\left(S_{0}(\tau) \phi\right) d \tau, & t+\theta>0, \\
\phi(t+\theta), & t+\theta \leq 0,\end{cases} \\
& =\left(S_{0}(t) \phi\right)(\theta)+X(t+\theta) \phi(0)+\int_{0}^{t+\theta} X(t+\theta-\tau) L\left(S_{0}(\tau) \phi\right) d \tau,
\end{aligned}
$$

where $X(t)$ is the fundamental operator of (1.1) (see [1]).

PROOF. Let

$$
\|T(t) \phi\|_{B}, \quad\|S(t) \phi\|_{B} \leq M e^{\eta t}, \quad t \geq 0, \quad \phi \in B,
$$

where $M>0, \eta>\omega$ (the constant in [1, Lemma 2.2]) are constants. For $\phi \in B$, set

$$
y(t)=y(t, \phi)= \begin{cases}0, & t>0 \\ \phi(t), & t \leq 0\end{cases}
$$

and $z(t)=z(t, \phi)=x(t, \phi)-y(t, \phi)$, where $x(t, \phi)$ is the solution of (1.1). Then we have

$$
\frac{d x}{d t}=L\left(z_{t}+S_{0}(t) \phi\right)=L\left(z_{t}\right)+L\left(S_{0}(t) \phi\right), \quad t>0 .
$$

By virtue of (H5) and (2.4), we obtain

$$
\left\|z_{t}\right\|_{B}, \quad\left\|S_{0}(t) \phi\right\|_{B} \leq M_{1} M e^{\eta t}, \quad t>0, \quad \phi \in B .
$$

Moreover, according to $(\mathrm{H} 2)$ or $(2.5)$,

$$
\|z(t)\|<M_{2} e^{\eta t}, \quad t>0,
$$

where $M_{2}>0$ is a constant. Thus, taking Laplace transform on two sides of the equality (2.5), we have that for $\operatorname{Re} \lambda>\eta$,

$$
-\phi(0)+\lambda \widehat{z}(\lambda)=L\left(e^{\lambda \cdot}\right) z(\lambda)+\int_{0}^{\infty} e^{-\lambda t} L\left(S_{0}(t) \phi\right) d t
$$

that is

$$
\widehat{z}(\lambda)=\left(\lambda I-L\left(e^{\lambda \cdot}\right)\right)^{-1} \phi(0)+\int_{0}^{\infty} e^{-\lambda t}\left(\lambda I-L\left(e^{\lambda \cdot}\right)\right)^{-1} L\left(S_{0}(t) \phi\right) d t,
$$

where $\widehat{z}(\lambda)$ is a Laplace transform of $z$. Accordingly, the formula (2.2) follows from the definition of $X(t)$, the convolution property and the uniqueness property of Laplace transform.

THEOREM 1. The solution semigroup $\{T(t)\}_{t \geq 0}$ of (1.1) is exponentially stable if and only if the fundamental operator $X(t)$ of (1.1) is exponentially stable and the phase space $B$ is an exponentially fading memory phase.

PROOF. Necessity. Let

$$
\|T(t) \phi\|_{B} \leq M_{0} e^{-\sigma t}\|\phi\|_{B} \text { for } t \geq 0, \phi \in B,
$$

where $M_{0}, \sigma>0$ are constants. Then, by (H5)

$$
\left\|S_{0}(t) \phi\right\|_{t}=\left\|T_{0}(t) \phi\right\|_{t} \leq\|T(t) \phi\|_{B} \leq M_{0} e^{-\sigma \tau}\|\phi\|, t \geq 0, \phi \in B,
$$

that is, $B$ is an exponentially fading memory space.

According to [1, Lemma 3.1], for every $b \in E, X(t) b$ satisfies

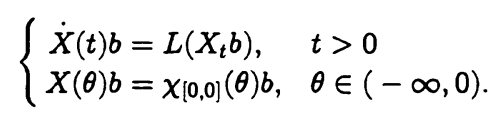

Therefore, 


$$
\left.\left(T(t) \chi_{[0,0]}(\cdot) b\right)(\theta)\right|_{\theta=0}=b+\int_{0}^{t} L\left(X_{\tau} b\right) d \lambda=X(t) b, b \in E .
$$

Consequently, it follows from (H2), (2.8) and (H6) that for any $b \in E$,

$$
\|X(t) b\| \leq K\left\|T(t) \chi_{[0,0]}(\cdot) b\right\| \leq K M_{0} M e^{-\sigma t}\|b\|, \quad t \geq 0 .
$$

This shows that $X(t)$ is exponentially stable.

Sufficiency. Obviously, we can suppose

$$
\begin{gathered}
\|X(t)\| \leq M_{0} e^{-\sigma t}(t \geq 0), \\
\left\|S_{0}(t) \phi\right\|_{B} \leq M_{0} e^{-\sigma t}\|\phi\|_{B}(t>0, \phi \in B) .
\end{gathered}
$$

Hence, by (H2),

$$
\sup _{-\frac{t}{2} \leq \theta<0}\|X(t+\theta) \phi(0)\| \leq M_{0} e^{-\frac{\sigma}{2} t}\|\phi(0)\| \leq M_{0} e^{-\frac{\sigma}{2} t}\|\phi\|_{B} .
$$

Moreover, for $\theta \in\left[-\frac{t}{2}, 0\right]$,

$$
\begin{aligned}
\left\|\int_{0}^{t+\theta} X(t+\theta-\tau) L\left(S_{0}(\tau) \phi\right) d \tau\right\| & \leq \int_{0}^{t+\theta} M_{0} e^{-\sigma(t+\theta-\tau)}\|L\| M_{0} e^{-\sigma \tau}\|\phi\|_{B} d \tau \\
& \leq M_{0}^{2}\|L\|(t+\theta) e^{-\sigma(t+\theta)}\|\phi\|_{B} .
\end{aligned}
$$

Accordingly,

$$
\sup _{-\frac{t}{2} \leq \theta \leq 0}\left\|\int_{0}^{t+\theta} X(t+\theta-\tau) L\left(S_{0}(\tau) \phi\right) d \tau\right\| \leq \frac{1}{2} M_{0}^{2}\|L\| t e^{-\frac{\theta}{2} t}\|\phi\|_{B}
$$

(H4), Lemma 1, (2.10) and (2.11) imply that

$$
\begin{aligned}
& \|T(t) \phi\|_{\left(\frac{t}{2}\right)} \leq K_{1} \sup _{-\frac{t}{2} \leq \theta \leq 0}\|(T(\tau) \phi)(\theta)\| \leq K_{1} \sup _{-\frac{t}{2} \leq \theta \leq 0}\|X(t+\theta) \phi(0)\|+ \\
& K_{1} \sup _{-\frac{1}{2} \leq \theta \leq 0}\left\|\int_{0}^{t+\theta} X(t+\theta-\tau) L\left(S_{0}(\tau) \phi\right) d \tau\right\| \leq\left(K_{1} M_{0}+\frac{1}{2} M_{0}^{2}\|L\| t\right) e^{-\frac{\theta}{2} t}\|\phi\|_{B} .
\end{aligned}
$$

As a consequence, there is a constant $K_{2}$ such that

$$
\|T(t) \phi\|_{\left(\frac{t}{2}\right)} \leq K_{2} e^{-\frac{2}{4} t}\|\phi\|_{B},
$$

taking into account $\lim _{t \rightarrow \infty} t e^{-\frac{l}{4} t}=0$.

On the other hand,

$$
\begin{aligned}
\|T(t) \phi\|_{\frac{t}{2}}=\left\|T\left(\frac{t}{2}\right)\left(T\left(\frac{t}{2}\right)\right) \phi\right\|_{\frac{t}{2}} & =\left\|S_{0}\left(\frac{t}{2}\right)\left(T\left(\frac{t}{2}\right) \phi\right)\right\|_{\frac{t}{2}} \\
& \leq\left\|S_{0}\left(\frac{t}{2}\right)\left(T\left(\frac{t}{2}\right) \phi\right)\right\| \leq M_{0} e^{-\frac{\sigma}{2} t}\left\|T\left(\frac{t}{2}\right) \phi\right\|_{B}
\end{aligned}
$$

Therefore, it follows from (H4), (2.12) and (2.13) that

$$
\|T(t) \phi\|_{B} \leq\|T(t) \phi\|_{\left(\frac{t}{2}\right)}+\|T(t) \phi\|_{\frac{t}{2}} \leq K_{2} e^{-\frac{q}{4} t}\|\phi\|_{B}+M_{0} e^{-\frac{q}{4} t}\left\|T\left(\frac{t}{2}\right) \phi\right\|_{B} .
$$

Consequently,

$$
\|T(t)\| \leq C e^{-\frac{g}{4} t}\left(1+\left\|T\left(\frac{t}{2}\right)\right\|\right)
$$


where $C=K_{2}+M_{0}$.

We say that $\|T(t)\|$ is bounded on $[0, \infty]$. In fact, if it is false, then as shown in [5],

$$
\omega_{L}=\inf _{t>0} \frac{\ln \|T(t)\|}{t} \geq 0
$$

that is, $\|T(t)\| \geq 1$. Hence, by (2 14),

$$
\begin{aligned}
\frac{\ln \|T(t)\|}{t} & \leq-\frac{\sigma}{4}+\frac{\ln C\left(1+\left\|T\left(\frac{t}{2}\right)\right\|^{-1}\right)}{t}+\frac{\ln \left\|T\left(\frac{t}{2}\right)\right\|}{t} \\
& \leq-\frac{\sigma}{4}+\frac{\ln 2 C}{t}+\frac{1}{2} \frac{\ln \left\|T\left(\frac{t}{2}\right)\right\|}{\frac{t}{2}} .
\end{aligned}
$$

Letting $t \rightarrow \infty$, we obtain

$$
\omega_{L} \leq-\frac{\sigma}{4}+\frac{1}{2} \omega_{L}
$$

i.e., $\omega_{L} \leq-\frac{\sigma}{2}$, which contradicts (2.15). So, $\|T(t)\|$ is bounded on $[0, \infty)$. Accordingly, (2.14) implies that $T(t)$ is exponentially stable.

REMARK 1. (i) If $L\left(S_{0}(t) \phi\right)$ is well defined for each $t>0, \phi \in B$, and

$$
L\left(\int_{0}^{\infty} e^{-\lambda t} S_{0}(t) \phi d t\right)=\int_{0}^{\infty} e^{-\lambda t} L\left(S_{0}(t) \phi\right) d t
$$

for $\operatorname{Re} \lambda>\omega$ ( $\omega$ is a constant), then Lemma 1 still holds when the hypothesis (H6) on $B$ is taken off

(ii) If the assumptions of (i) hold, $\left\|L\left(S_{0}(t) \phi\right)\right\| \leq M\left\|S_{0}(t) \phi\right\|_{t}$ for any $t>0, \phi \in B$ and some constant $M>0$, and $E$ is a finite-dimensional space, then Theorem 1 is still true when the hypothesis (H6) on $B$ is taken off.

PROOF. (i) and the "sufficiency" of (ii) are clearly true according to the proof of Lemma 1 and that of the "sufficiency" of Theorem 1 respectively.

Let us look at the "Necessity" of (ii) now. Obviously, by (2.9), $B$ is an exponentially fading memory space. From (2.8), $\omega_{L}=\inf _{t>0} \frac{\ln \|T(t)\|}{t} \leq-\sigma$. Accordingly, for $\operatorname{Re} \lambda>-\sigma, b \in E, e^{\lambda \theta} b \in B$ and $\operatorname{det}\left(\lambda-L\left(e^{\lambda \cdot}\right)\right) \neq 0$, as shown in [3]. Taking a $\sigma_{0} \in(0, \sigma)$, we have by (H5) that $\left\|e^{\lambda \cdot b}\right\|_{B} \leq M_{1} \| e^{-\sigma_{0} \cdot b \|_{B}}$ and $\| L\left(e^{\lambda \cdot b} b\|\leq\| L\left\|M_{1}\right\| e^{-\sigma} \cdot b \|_{B}\right.$ for $R e \lambda \geq-\sigma_{0}$. Thanks to the resonance theorem, we get $\sup \left\{\left\|L\left(e^{\lambda \cdot}\right)\right\|: \operatorname{Re} \lambda \geq-\sigma_{0}\right\}<\infty$. Therefore, $X(t)$ is exponentially stable by virtue of [1, Theorem 3.5$]$.

ACKNOWLEDGMENT. This work was supported by the National NSF of China and the ABSF of Yunnan Province. The authors are very grateful to the referee for his careful reading and valuable suggestions.

\section{REFERENCES}

[1] LIANG, J. and XIAO, T.J., Functional differential equations with infinite delay in Banach spaces, Inter. J. Math. \& Math. Sci. 14 (1991), 497-508.

[2] CORDUNEANU, C. and LAKSHMIKANTHAM, V., Equations with unbounded delay: A survey, Nonlinear Analysis Theory Method and Application 4 (1980), 831-877.

[3] NAITO, T., On linear autonomous retarded equations with an abstract phase space for infinite delay, J. Diff. Eqns. 33 (1979), 74-91.

[4] HALE, J.K and KATO, J., Phase space for retarded equations with infinite delay, Funcl. Ekvaciaj. 21 (1978), 11-41.

[5] YOSIDA, K., Functional Analysis, Springer-Verlag, Berlin, Heidelberg, 1965. 


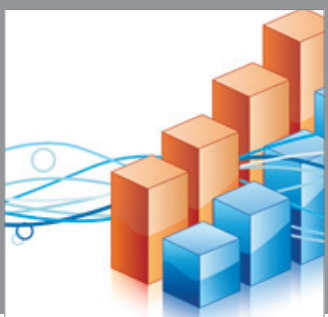

Advances in

Operations Research

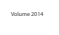

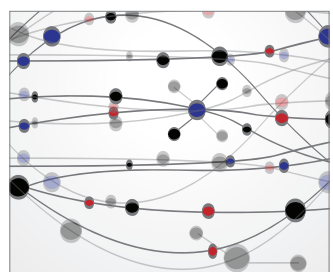

\section{The Scientific} World Journal
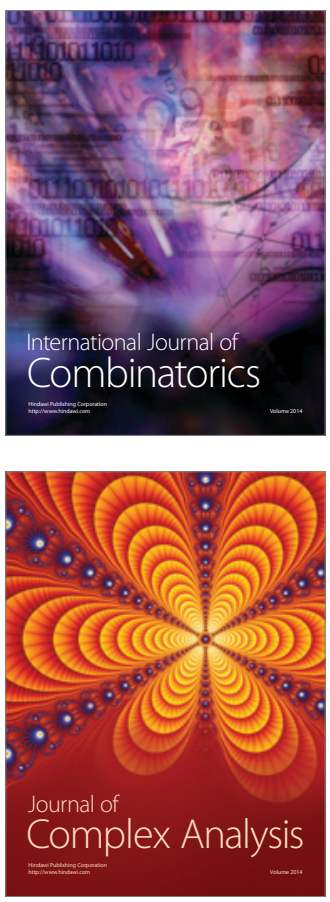

International Journal of

Mathematics and

Mathematical

Sciences
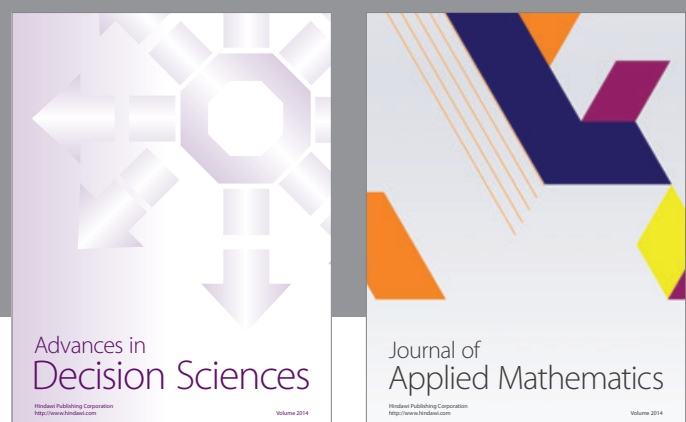

Journal of

Applied Mathematics
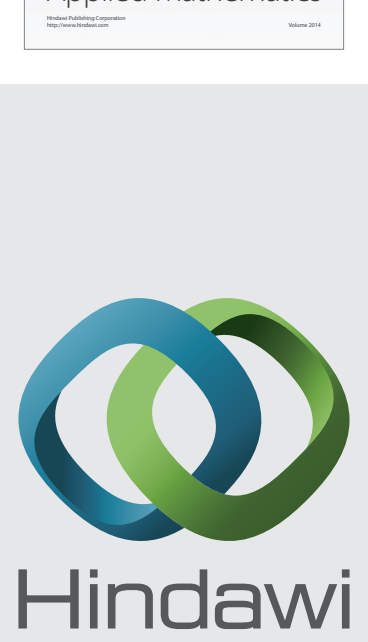

Submit your manuscripts at http://www.hindawi.com
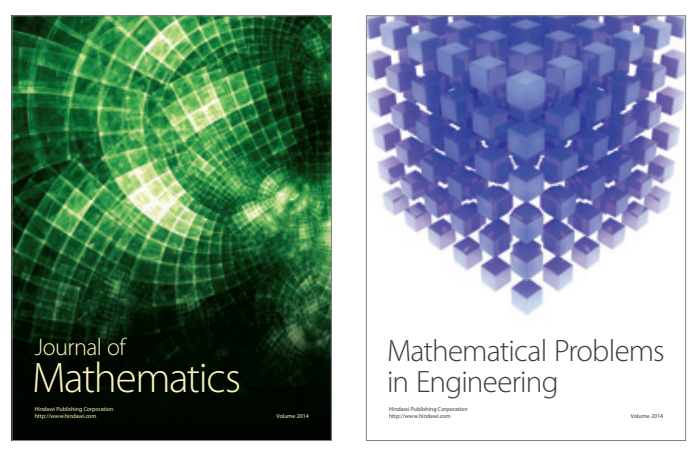

Mathematical Problems in Engineering
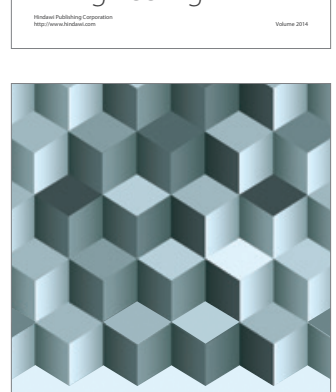

Journal of

Function Spaces
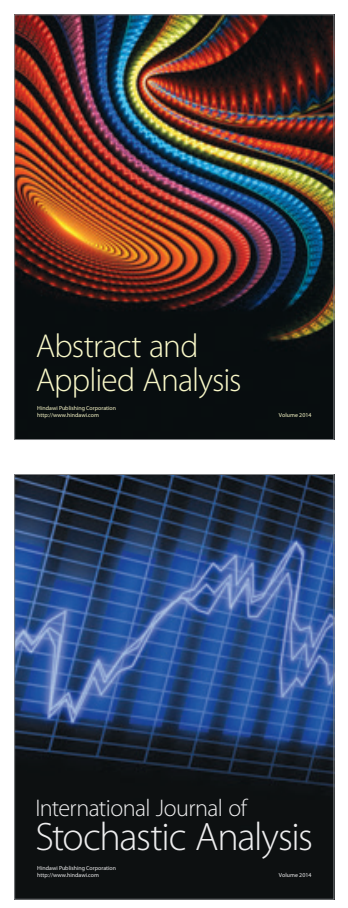

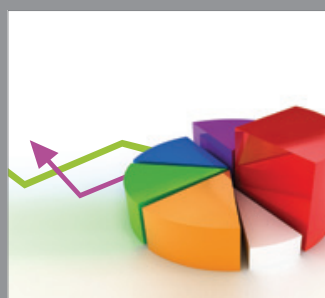

ournal of

Probability and Statistics

Promensencen
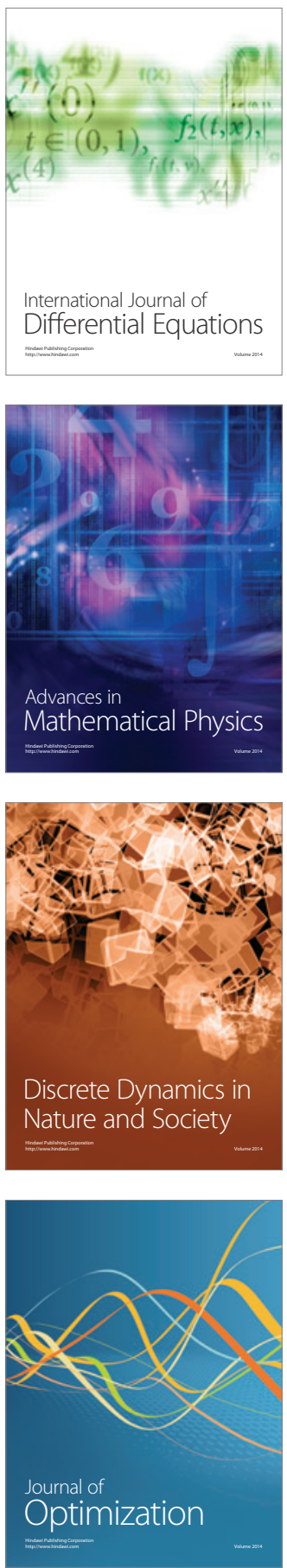